\title{
La bibliothèque de Johann Philipp von Wessenberg- Ampringen : un don fondateur de l'identité européenne de la BNU
}

Peter Heinrich von WessenbergDescendant direct de Johann Philipp von Wessenberg-Ampringen, Peter Heinrich von Wessenberg est notamment le fondateur de la Wessenberg-Akademie, une plateforme et un forum de discussion sur l'histoire des régions du Rhin et du Danube (www.wessenberg.at) [Note du traducteur]., Roger MassieNé en Inde et ancien fonctionnaire au Conseil de l'Europe, Roger Massie - qui réside aujourd'hui à Kolbsheim, en Alsace - est comme son cousin Peter Heinrich von Wessenberg le descendant de Johann Philipp von Wessenberg. Le texte publié ici est une version largement revue et augmentée d'une conférence donnée en 2004 avec le concours et les recherches dili Gaspard, lors de la 3e réunion annuelle de la WessenbergAkademie, à Liebenswiller et en Alsace [note du traducteur].

Traducteur : Julien Collonges

\section{(2) OpenEdition Journals}

Édition électronique

URL : http://journals.openedition.org/rbnu/1286

DOI : $10.4000 /$ rbnu. 1286

ISSN : 2679-6104

Éditeur

Bibliothèque nationale et universitaire de Strasbourg

Édition imprimée

Date de publication : 1 novembre 2015

Pagination : 8-19

ISBN : 9782859230616

ISSN : $2109-2761$

Référence électronique

Peter Heinrich von WessenbergDescendant direct de Johann Philipp von Wessenberg-Ampringen, Peter Heinrich von Wessenberg est notamment le fondateur de la Wessenberg-Akademie, une plateforme et un forum de discussion sur l'histoire des régions du Rhin et du Danube (www.wessenberg.at) [Note du traducteur]., Roger MassieNé en Inde et ancien fonctionnaire au Conseil de l'Europe, Roger Massie - qui réside aujourd'hui à Kolbsheim, en Alsace - est comme son cousin Peter Heinrich von Wessenberg le descendant de Johann Philipp von Wessenberg. Le texte publié ici est une version largement revue et augmentée d'une conférence donnée en 2004 avec le concours et les recherches dili Gaspard, lors de la 3e réunion annuelle de la Wessenberg-Akademie, à Liebenswiller et en Alsace [note du traducteur]., « La bibliothèque de Johann Philipp von WessenbergAmpringen : un don fondateur de l'identité européenne de la BNU », La Revue de la BNU [En ligne], 12 I 2015, mis en ligne le 01 mars 2020, consulté le 16 décembre 2020. URL : http://

journals.openedition.org/rbnu/1286; DOI : https://doi.org/10.4000/rbnu.1286 


\section{(c) (1) $\odot(-)$}

La Revue de la BNU est mise à disposition selon les termes de la Licence Creative Commons

Attribution - Pas d'Utilisation Commerciale - Partage dans les Mêmes Conditions 4.0 International. 


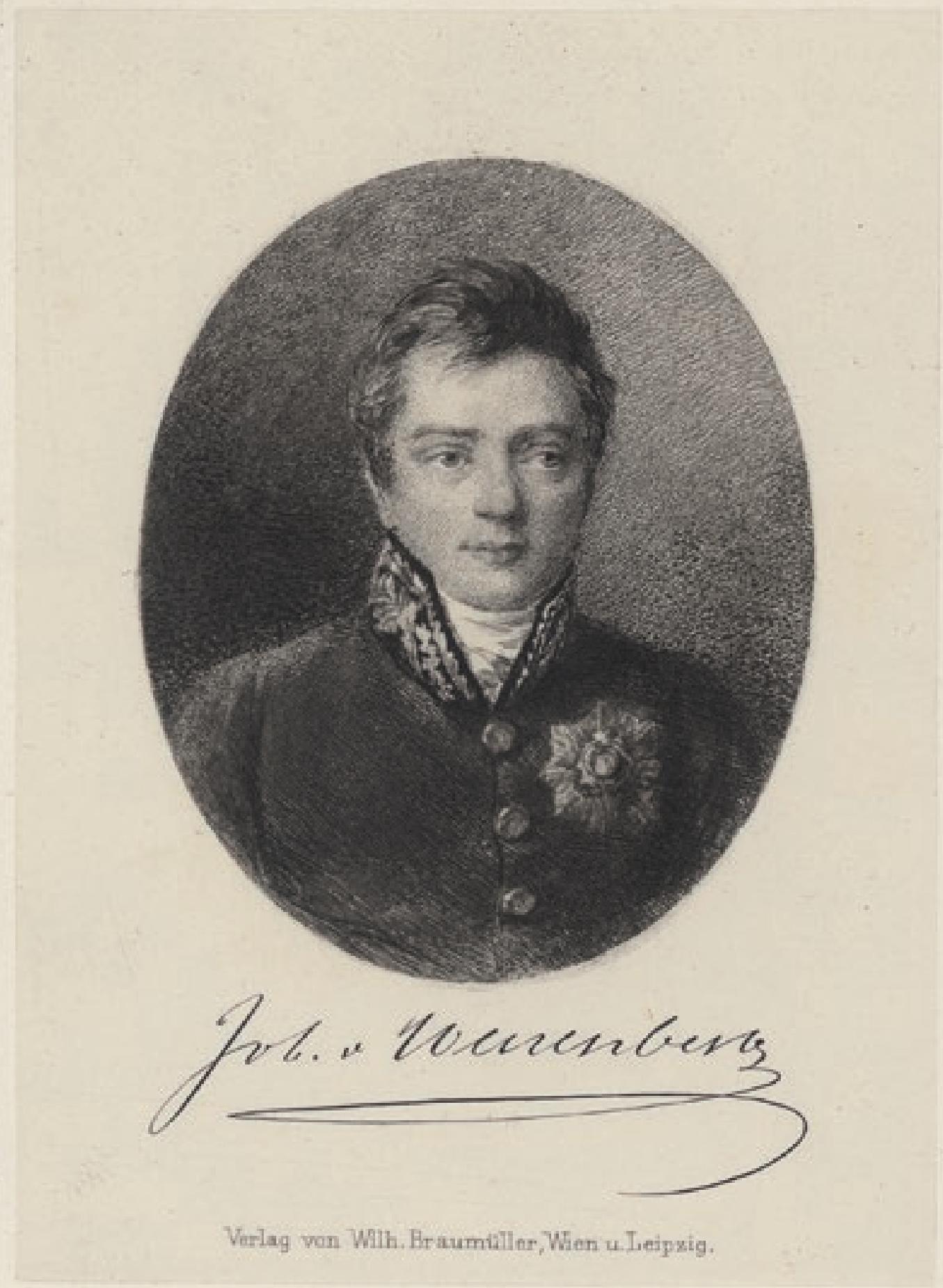

Portrait gravé de Johann Philipp von Wessenberg-Ampringen dans la biographie que lui a consacré Alfred von Arneth en 1898 (Johann Freiherr von Wessenberg: ein österreichischer Staatsmann des neunzehnten Jahrhunderts, Wien, W. Braumüller ; coll. BNU). 


\title{
La bibliothèque de Johann Philipp von Wessenberg-Ampringen :
}

\author{
un don fondateur de l'identité européenne de la BNU
}

\section{Avant-propos}

\section{Comment éclairer la personnalité de celui qui se cache derrière la collection de livres?}

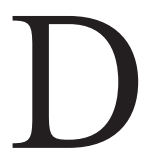

ans le cas du baron Johann Philipp von Wessenberg, il est particulièrement important de tenir compte de la situation géopolitique de son temps. Il descend d'une très ancienne famille noble originaire de la Burgunderpforte, ou Porte du Sundgau. Le château familial ainsi que les premiers biens allodiaux et les premiers fiefs étaient situés dans le canton suisse d'Argovie, à proximité des communes de Mandach et Hottwil im Aargau, et dès le $11^{\mathrm{e}}$ siècle la famille était en relation avec le célèbre monastère bénédictin de Säckingen, dont les possessions s'étendaient de part et d'autre des rives du Rhin, jusqu'à Laufenburg et audelà. Au cours des siècles qui suivirent, les Wessenberg se rangèrent toujours aux côtés des Habsbourg, mais aussi des princes-évêques de Bâle. Des uns et des autres, ils obtinrent des fiefs, avec des châteaux et fermes seigneuriales, qui se situaient en Alsace, dans l'actuel canton de Bâle et dans le Brisgau. C'est pour désigner les terres des Wessenberg et des Habsbourg que le terme d'“ Autriche antérieure » (Vorderösterreich) fut forgé. On peut donc dire que les frontières de la famille Wessenberg étaient constamment mouvantes. Par le jeu des alliances familiales, fréquentes à l'époque, les zones d'implantation des chevaliers, des diplomates ou des hommes d'Église (la " noblesse chapitrable ") incluaient de vastes territoires dans le Haut-Rhin et le Rhin supérieur. Jusqu'au début du $19^{\mathrm{e}}$ siècle, ces territoires - à l'exception de la principauté épiscopale de Bâle - étaient dominés par les Habsbourg
d'Autriche. À partir du $18^{\mathrm{e}}$ siècle, en dehors de leurs possessions seigneuriales déjà existantes dans l'Alsace française, dans plusieurs régions suisses et dans les principautés de Bade et du Wurtemberg, les barons von Wessenberg apparaissent aussi comme propriétaires de châteaux et de biens en Bohême et en Hongrie, et occupent des positions dominantes comme diplomates ou maréchaux au sein de la cour du roi de Saxe et de Pologne.

Le plus important représentant de la lignée est Ignaz Heinrich von Wessenberg-Ampringen, dernier évêque de la principauté épiscopale de Constance (dissoute en 1828), qui se fit un nom comme humaniste, écrivain, collectionneur d'art et mécène (sa très riche bibliothèque fut léguée en ma présence, en 2001, à l'Université de Constance). Il fait aujourd'hui encore l'objet de beaucoup d'intérêt dans des écrits publiés en Suisse et en Allemagne.

Son frère aîné, personnalité non moins éminente à son époque, est le baron Johann Philipp von WessenbergAmpringen ${ }^{1}$ qui fut second négociateur plénipotentiaire pour l'Autriche au Congrès de Vienne en 1814/15, ministre plénipotentiaire lors de la Conférence de Londres en 1834, diplomate actif dans toutes les grandes capitales des régions allemandes de l'époque, de la Bavière à la Prusse, ainsi qu'au parlement fédéral de Francfort, et qui fut même appelé, au soir de sa vie en 1848 , à exercer les plus hautes fonctions de ministre des Affaires étrangères et de ministre-président de l'Autriche à la suite de Metternich. Durant sa vie, il fut lié, par des liens de conseil et d'amitié, à certaines des personnalités les plus éminentes et les plus intéressantes de l'époque, comme le premier roi de Belgique Léopold I ${ }^{\text {er }}$ ou l'archiduc Jean-Baptiste d'Autriche. 
Par ces quelques éléments, nous espérons avoir brièvement restitué la dimension " mitteleuropéenne " de Johann Philipp von Wessenberg-Ampringen, l'un des diplomates autrichiens ayant servi le plus longtemps, et dont la bibliothèque et les penchants bibliophiles vont être ici présentés plus longuement.

\section{Peter Heinrich von Wessenberg ${ }^{2}$}

(traduction Julien Collonges)

$\star \star \star$

Je suis, comme mon cousin Peter Heinrich von Wessenberg, l'arrière-arrière-arrière-arrière-petit-fils de l'éminent homme d'État autrichien Johann Phillip von Wessenberg - qui fut l'un des proches collaborateurs du prince de Metternich lors du Congrès de Vienne et qui devint même brièvement son successeur en 1848, l'année de plusieurs révolutions européennes. Mes recherches sur sa bibliothèque qui, pour des raisons historiques, est aujourd'hui conservée à la BNU de Strasbourg, ont semble-il suscité quelque intérêt.

Comme on le sait, la bibliothèque du Séminaire protestant de Strasbourg - qui faisait alors office de bibliothèque universitaire - et la bibliothèque de la Ville de Strasbourg, toutes deux réunies au Temple Neuf, furent détruites lors du siège de la ville en 1870 . Le 27 décembre 1876, dix-huit ans après la mort de Johann Philipp von Wessenberg, un général prussien, le comte Clotar Blankensee-Fircks zu Wugarten, nouveau propriétaire de la dernière demeure de Johann Philipp, le château de Feldkirch dans le pays de Bade, fit don de l'intégralité de la bibliothèque de l'homme d'État autrichien à la nouvelle Kaiserliche Universitätsund Landesbibliothek zu Straßburg, fondée par les autorités allemandes en réparation de la destruction des bibliothèques strasbourgeoises.

Les titres des ouvrages qui composent cette remarquable bibliothèque parlent par eux-mêmes, davantage qu'ils ne reflètent la biographie de celui qui fut ministre-président de l'Autriche. D'après l'inventaire de la bibliothèque, conservé dans les archives de la $\mathrm{BNU}^{3}$, le fonds est constitué de 2157 titres (correspondant à 3786 volumes), écrits dans des langues très diverses. Presque la moitié d'entre eux est en langue française et environ un quart en langue allemande. Viennent ensuite, par ordre d'importance, les ouvrages en anglais, en italien, en latin et en espagnol.
Dans une moindre mesure enfin, l'inventaire comprend aussi quelques livres en hollandais, en grec, en portugais, en hébreu, et même en tchèque.

Pour mon étude, je me suis d'abord intéressé, en première approche, au seul corpus des ouvrages en langue allemande, dont les titres sont signalés dans l'inventaire en écriture cursive allemande du $19^{\mathrm{e}}$ siècle. Si les résultats ainsi obtenus ne peuvent être étendus comme tels à l'ensemble du fonds, ils constituent toutefois un faisceau d'indications intéressantes sur la nature et la composition de la bibliothèque.

Un regard jeté sur ces titres en langue allemande permet tout d'abord de mettre en évidence des données intéressantes sur l'âge et l'origine des livres qui composent la bibliothèque. Une partie importante (presque $12 \%$ ) des quelque 650 volumes en langue allemande provient de Leipzig ; viennent ensuite les livres édités à Vienne $(8,7 \%)$, à Berlin $(7 \%)$ et Francfort $(3,7 \%)$. Le reste des ouvrages en langue allemande a été publié dans de nombreuses autres villes d'Allemagne, de Prusse, d'Autriche-Hongrie, de Suisse, de Russie... et même d'Égypte ! Les dates de publication varient, elles, entre le $16^{\mathrm{e}}(1522$ pour le plus ancien) et le $19^{\mathrm{e}}$ siècles, même si, en y regardant de plus près, l'essentiel des ouvrages a été publié entre 1800 et 1860 . Cela signifie que dans chacune des disciplines et thématiques représentées, Johann Philipp s'est efforcé de bâtir une collection incluant les ouvrages les plus récents de son époque.

D'un point de vue thématique, il apparaît que le corpus des livres en langue allemande comprend un nombre considérable d'œuvres de nature historique (141 titres, soit plus de $20 \%$ des titres en allemand) relevant principalement de l'histoire contemporaine. La politique et le droit viennent au second rang, avec respectivement 73 et 56 titres (11 et $9 \%$ ). À noter également le nombre important de monographies (plus de 50 , soit $8 \%$ ), de biographies et de volumes de correspondance (40 titres, 6\%), d'ouvrages de géographie et de statistique (respectivement 40 et 33 titres, soit 6 et 5\%), ainsi que la présence d'au moins 59 œuvres (9\%) relatives à la finance, à la monnaie, au commerce et à la politique économique. Les guides et livres de voyages (36 titres, 5,5\%) ne sont pas oubliés, tout comme plusieurs volumes relatifs à l'art des jardins et à l'art de la guerre. Enfin, de nombreuses autres disciplines et thématiques sont également représentées : la littérature, l'art, les sciences de la nature, la rhétorique, la morale, la religion, la pédagogie, la sociologie, les affaires administratives, la philosophie, etc. 


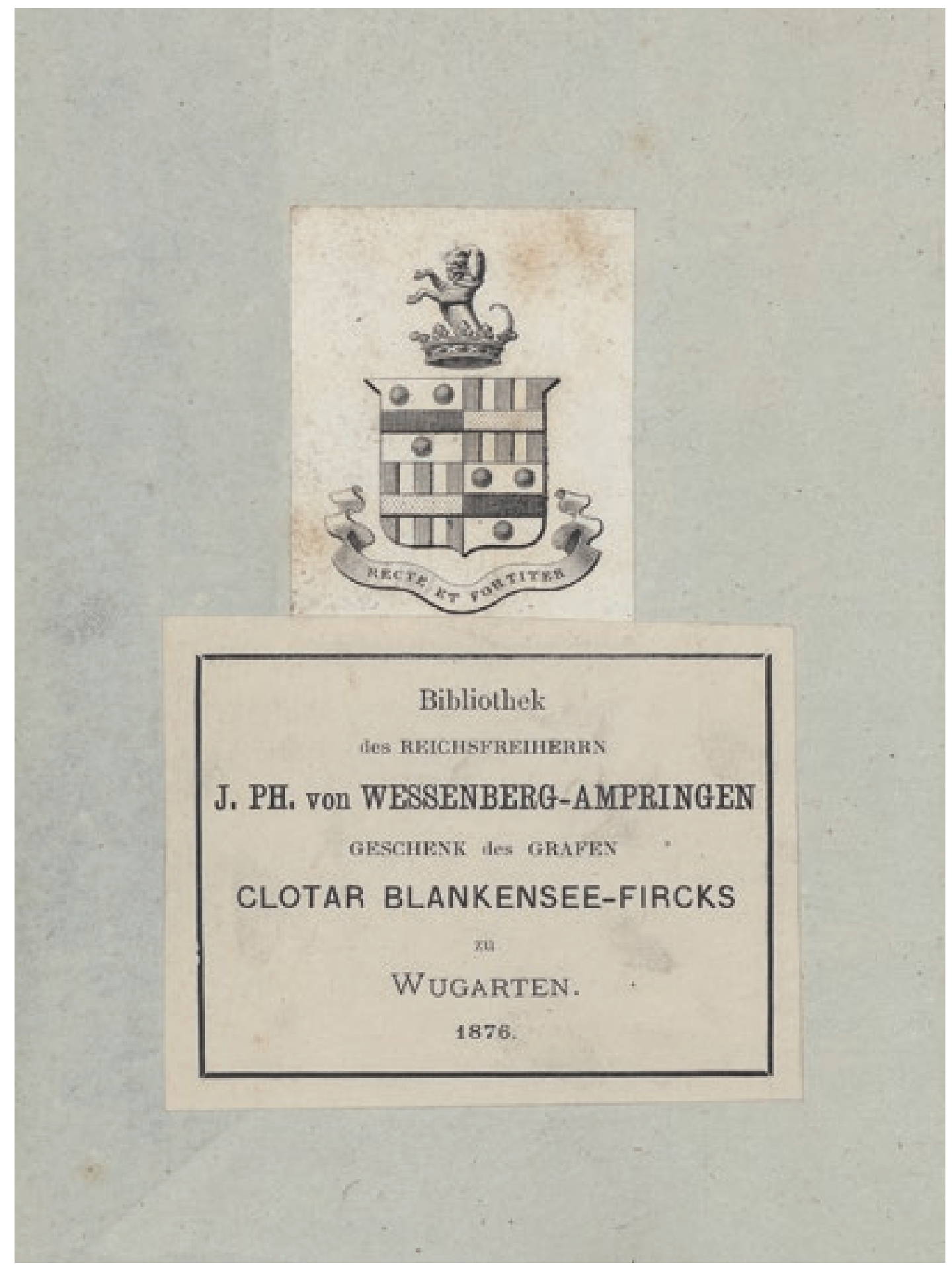




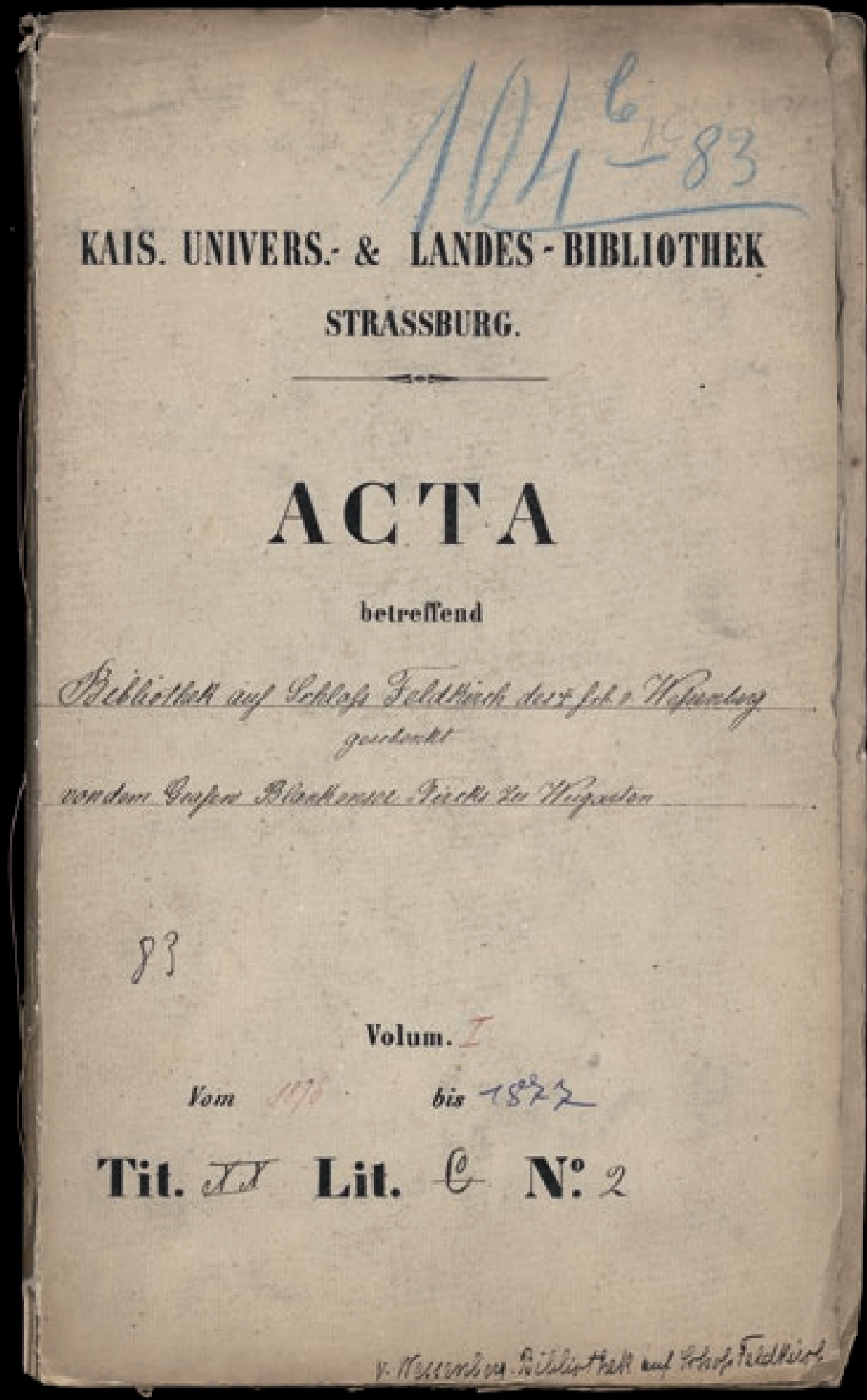

Dossier des archives de la BNU contenant les pièces relatives au don de la bibliothèque de Johnann Philipp von Wessenberg-Ampringen (coll. BNU) 
Mais au-delà de ces données très générales, que nous dit le contenu de l'ancienne bibliothèque du château de Feldkirch sur l'homme que fut Johann Philipp von Wessenberg - et pas seulement sur le négociateur plénipotentiaire du Congrès de Vienne, Grand-croix de l'Ordre de Saint-Étienne de Hongrie ? Voici quelques-unes des remarques, plus subjectives qu'académiques, que m’a inspirées l'étude de l'inventaire de sa bibliothèque.

Tout d'abord, on a le sentiment qu'il s'agit là de la bibliothèque d'un véritable ami des livres, même si l'un de ses ouvrages, The Library companion, or the Young man's guide and the old man's comfort, in the choice of a library (1824) pourrait laisser accroire - à tort - que cet amour n'a rien de spontané. Plusieurs ouvrages de sa bibliothèque confirment du reste les penchants bibliophiles de Johann Philipp, notamment les dictionnaires et catalogues analytiques du célèbre bibliographe français AntoineAlexandre Barbier, qui ont pu lui servir de guide dans la constitution de sa collection.

Ce qui frappe ensuite, c'est que si la bibliothèque de mon aïeul comprend d'innombrables titres relatifs à la politique et à l'histoire contemporaine et reflète ainsi très naturellement son activité de diplomate et d'homme d'État, ce corpus est bien loin d'épuiser les centres d'intérêt très variés qui furent les siens. Si l'on ne considère que les ouvrages qu'il a lui-même publiés et qui sont bien sûr présents dans la bibliothèque -, on constate qu'outre les études qu'il a consacrées à certaines questions politiques et économiques de son temps, comme la première guerre d'indépendance italienne (Übersicht des viertägigen Feldzuges in Italien, 1849) ou la situation financière de la France (Ein Blick in die Geschichte der französischen Finanz-Verwaltung, s. d.), il fut aussi l'auteur de plusieurs guides et récits de voyage (La Route du Simplon, 1812 ; Monuments at Munich, 1839 ; Souvenirs de voyage : Constance et ses environs, s. d.), d'un livre sur le mémorial du Walhalla et même d'un recueil d'Observations sur l'art d'écrire (s. d.). En définitive, c'est peut-être l'important fonds de ses manuscrits autographes - qui fut cédé à la BNU en même temps que sa bibliothèque qui reflète le plus étroitement son intense activité diplomatique et politique. Parmi les quinze recueils conservés à la BNU, on trouve ainsi notamment un Diarium politicum, des Mélanges d'économie politique, des Fragmens politiques (dans lesquels Johann Philipp analyse, en français, les causes de la Révolution française ou l'avènement de Napoléon ${ }^{4}$ ), et une importante et passionnante collection d'autographes contenant des documents de la main des plus importants diplomates et hommes d'État de son temps, de Charles-Joseph, duc de Lorraine, au comte de Nesselrode, en passant par le baron de Münchhausen, le prince Auguste de Prusse, Anne-Amélie de Brunswick et même Bonaparte luimême. Et pourtant, même ici, certains de ces manuscrits trahissent la diversité des centres d'intérêt de Johann Philipp : un recueil est intitulé Litterarische Erinnerungen, un autre Album litterarico-statisticum, 1830, et le recueil des autographes contient aussi une très intéressante lettre de Gotthold Ephraim Lessing à sa mère, datée du 20 janvier 1749, dans laquelle il raconte ses années de jeunesse à Leipzig.

$\mathrm{Du}$ reste, parmi les nombreux titres relatifs à la politique et à l'histoire contemporaine - qui constituent à eux seuls un véritable trésor -, j'ai été particulièrement frappé par le fait qu'à côté des très nombreux ouvrages de et sur Napoléon, ses généraux ou sa famille (citons par exemple cet assez rare Bonaparte, l'Armée et le Sénat, dialogue entre un Parisien et un paysan, publié par Napoléon en 1814, ou la Proclamation de Napoléon Buonaparte à ses nouveaux sujets. Constitution de l'isle d'Elbe parue la même année), de ceux consacrés à Talleyrand, à Fouché (comme la Lettre du duc d'Otrante au duc de Wellington publiée à Londres en 1816) ou à plusieurs hommes et femmes d'État britanniques (Henry Brougham, Henry Richard Vassall-Fox, Marie Stuart), on ne trouve quasiment aucun titre sur celui que Johann Philipp conseilla et auquel il succéda, le prince de Metternich. Un seul livre l'évoque, Les Diplomates européens de Jean-Baptiste Capefigue (1843), mais c'est au milieu d'une série d'autres grands hommes politiques européens. Pour le reste, il y a là les œuvres de certains des plus éminents hommes d'État et historiens français (Maurice de Saxe, Louis Blanc, François Guizot, Adolphe Thiers ou Jules Michelet, avec l'édition originale complète de son Histoire de la Révolution française et la deuxième édition du Peuple en 1846), de l'historien anglais David Hume, de Frédéric II de Prusse, de nombreux traités de paix et d'alliance entre les nations européennes et plusieurs ouvrages consacrés au Congrès de Vienne et au projet politique européen (comme le traité de Saint-Simon, De la réorganisation de la société européenne, paru en 1814, qui jette les bases d'une union politique européenne ${ }^{5}$ ). Les titres relatifs à l'économie et à la finance, eux aussi fort nombreux, ne concernent pas non plus directement l'Autriche. Ce sont pour la plupart des œuvres des plus grands théoriciens européens de l'économie : Necker (par exemple le traité 
De l'administration des finances de la France, édition de 1785, ou l'édition originale de son Mémoire justificatif de 1787), Malthus (la traduction française parue en 1820 de ses Principes d'économie politique), Turgot (ses œuvres complètes en neuf volumes parues en 1808-1811), Say (une édition de 1814 de son Traité d'économie politique), Ricardo (la première traduction française de l'ouvrage Des principes de l'économie politique et de l'impôt, 1819) ou Adam Smith (une traduction allemande parue en 1812 de ses Recherches sur la nature et les causes de la richesse des nations). Si bien qu'au total, à la lecture de l'inventaire, le lecteur dilettante que je suis a le sentiment qu'il s'agit moins là de la bibliothèque d'un homme politique autrichien de premier plan que de celle d'un « homme du monde cultivé ", européen et tourné vers le monde, à la fois francophile et anglophile, et affublé des goût parfois singuliers propre à l'aristocratique $18^{\mathrm{e}}$ siècle.

Que Johann Philipp von Wessenberg ait été un homme de culture, curieux de tout et ouvert sur le monde, c'est ce que montre tout d'abord sa passion des voyages, dont témoignent de multiples ouvrages conservés dans sa bibliothèque. Outre ceux qu'il publia lui-même, on y trouve d'innombrables guides et récits consacrés à des destinations européennes (principalement en Italie et au Royaume-Uni, mais également en Suisse, en Hollande, en Europe centrale et en Russie), comme aussi des titres évoquant des voyages au long cours (le Voyage de La Pérouse autour du monde de La Pérouse, dans l'édition de 1798, ou le Premier voyage autour du monde d'Antonio Pigafetta, édition de 1801) et des destinations plus exotiques (Chili, Pérou, Mexique, Colombie, Syrie, Égypte, etc.). Certains titres pourront être familiers aux lecteurs strasbourgeois, comme cette édition du Voyage littéraire en Alsace du bénédictin Thierry Ruinart (traduction française de 1829); d'autres excitent l'imagination et la curiosité, comme ces Voyages de Mirza Abu Taleb Khan, en Asie, en Afrique et en Europe, pendant les années 1799, 1800, 1801, 1802 et 1803 ; écrits par lui-même en persan. Suivis d'une réfutation des idées qu'on a en Europe sur la liberté des femmes d'Asie, publiés à Strasbourg en 1811.

Johann Philipp fut ensuite, et surtout, un fin lettré, familier des plus grands auteurs de la littérature et de la culture européennes. On trouve ainsi par exemple dans sa bibliothèque plusieurs œuvres des grands auteurs de l'Antiquité classique, souvent en langue originale : Cicéron, Pline le Jeune (avec une édition latine du livre $\mathrm{X}$ de ses Lettres publiée en 1650 à Francfort), Plutarque (une édition strasbourgeoise du De liberorum educatione commentarius parue en 1663), Virgile, Salluste, Suétone, Tacite ou encore Hérodote. Les classiques de la littérature moderne - principalement européenne - sont aussi présents en grand nombre : la littérature anglaise avec Byron (notamment la première traduction française de ses Mémoires, parue en 1825), Mary et Percy Bysshe Shelley (la première réédition, en 1829, de leur History of six weeks), Macaulay, Scott (les premières traductions françaises de Peveril du Pic, 1823, et de Woodstock, 1826), Milton, Trollope (la première traduction française de $L a$ Petite maison d'Allington, 1866), Walpole, Pope ou Charlotte Brontë (une édition anglaise de Jane Eyre publiée en 1850) ; la littérature espagnole avec Cervantès et italienne avec Boccace et Machiavel (une rare édition italienne de 1550 de ses Historie) ; la littérature allemande avec Goethe (l'édition originale allemande de Winckelmann et son siècle, $1805^{6}$ ), Schiller (notamment une édition allemande de Don Carlos parue en 1801), Heine (édition originale de Zur Geschichte der neueren schönen Literatur in Deutschland, 1833), Klopstock, Wieland (première édition de son roman Aristipp en 1800-1802) ou Kotzebue ; et même la littérature américaine avec Washington Irving (notamment le recueil, paru en 1824, des numéros de sa très curieuse revue satirique Salmagundi).

Mais c'est surtout le corpus des auteurs français qui est le plus impressionnant, avec des éditions en langue originale - parfois très rares - des plus éminents écrivains de toute l'histoire littéraire de la France : le $16^{\mathrm{e}}$ siècle humaniste est représenté par Montaigne et Rabelais (avec une belle édition en trois volumes de ses œuvres, illustrée par Charles Thomson et AlexandreJoseph Desenne) et le Grand Siècle par Racine (les Euvres complettes [sic] en sept volumes illustrées par JeanBaptiste Santerre, 1807), La Fontaine (avec une édition de 1721 des Contes et nouvelles en vers et une édition illustrée des Fables parue en 1780), Fénelon (l'édition originale des Directions pour la conscience d'un roi, 1747 ${ }^{7}$ ), La Bruyère et La Rochefoucauld (édition de 1714 des Réflexions, sentences et maximes morales). Le siècle des Lumières est aussi abondamment présent, avec des œuvres de Voltaire, Diderot (édition de 1799 de La Religieuse), Rousseau (édition de 1792 de La Nouvelle Héloïse), Montesquieu, Mirabeau, Grimm et La Harpe (édition originale de sa tragédie Coriolan, 1784), de Madame du Deffand, Mme d'Épinay et Mme de Genlis (édition originale de La Feuille des gens du monde, 1813, de ses Mémoires inédits, 1825, et des Soupers de la Maréchale de Luxembourg, 1828), de Chamfort, Chénier, Marmontel (édition de 1804 des Mémoires d'un père pour servir à l'éducation de ses enfants) et de Bernardin 


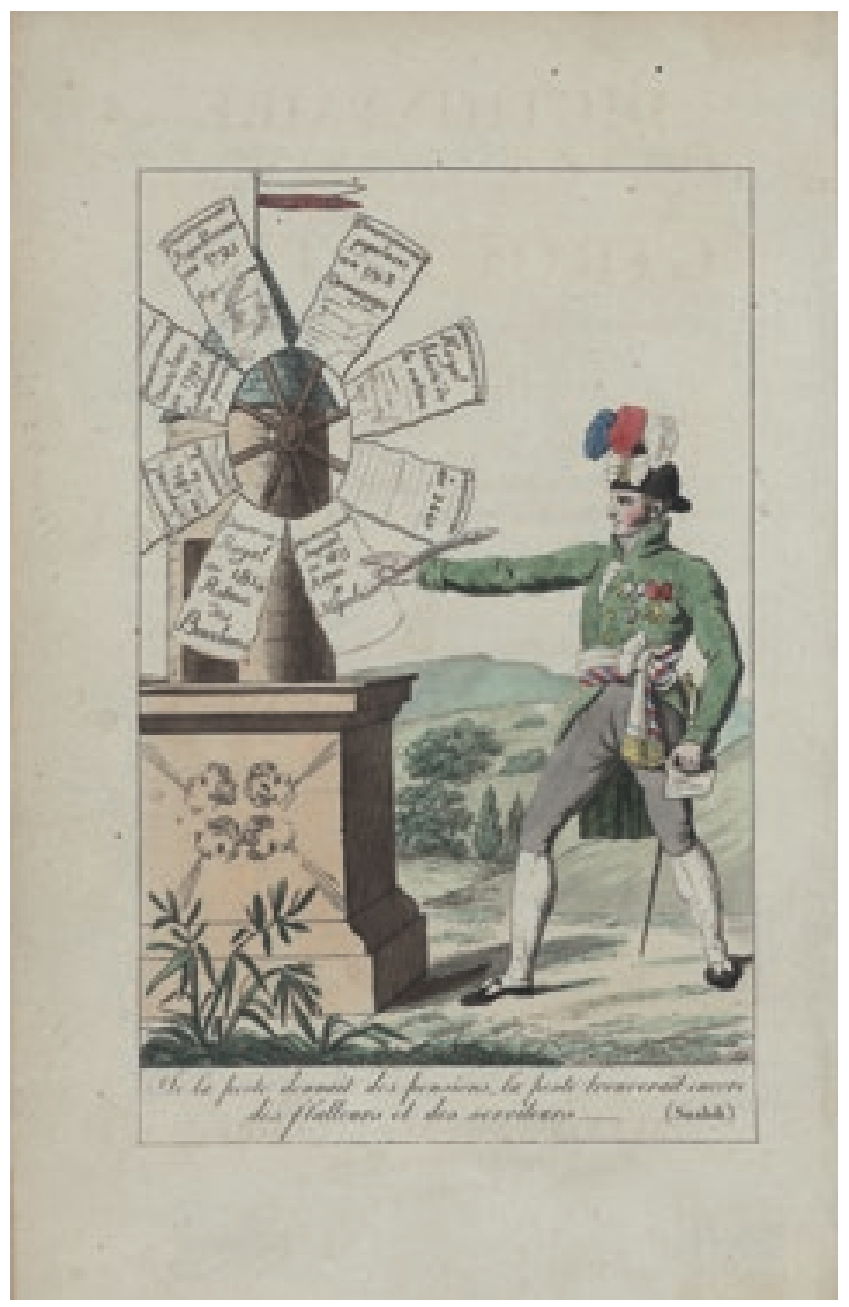

"Gravure allégorique " anonyme publiée au frontispice du Dictionnaire des girouettes, ou Nos contemporains peints d'après eux-mêmes (Paris, Alexis Eymery, 1815 ; coll. BNU)

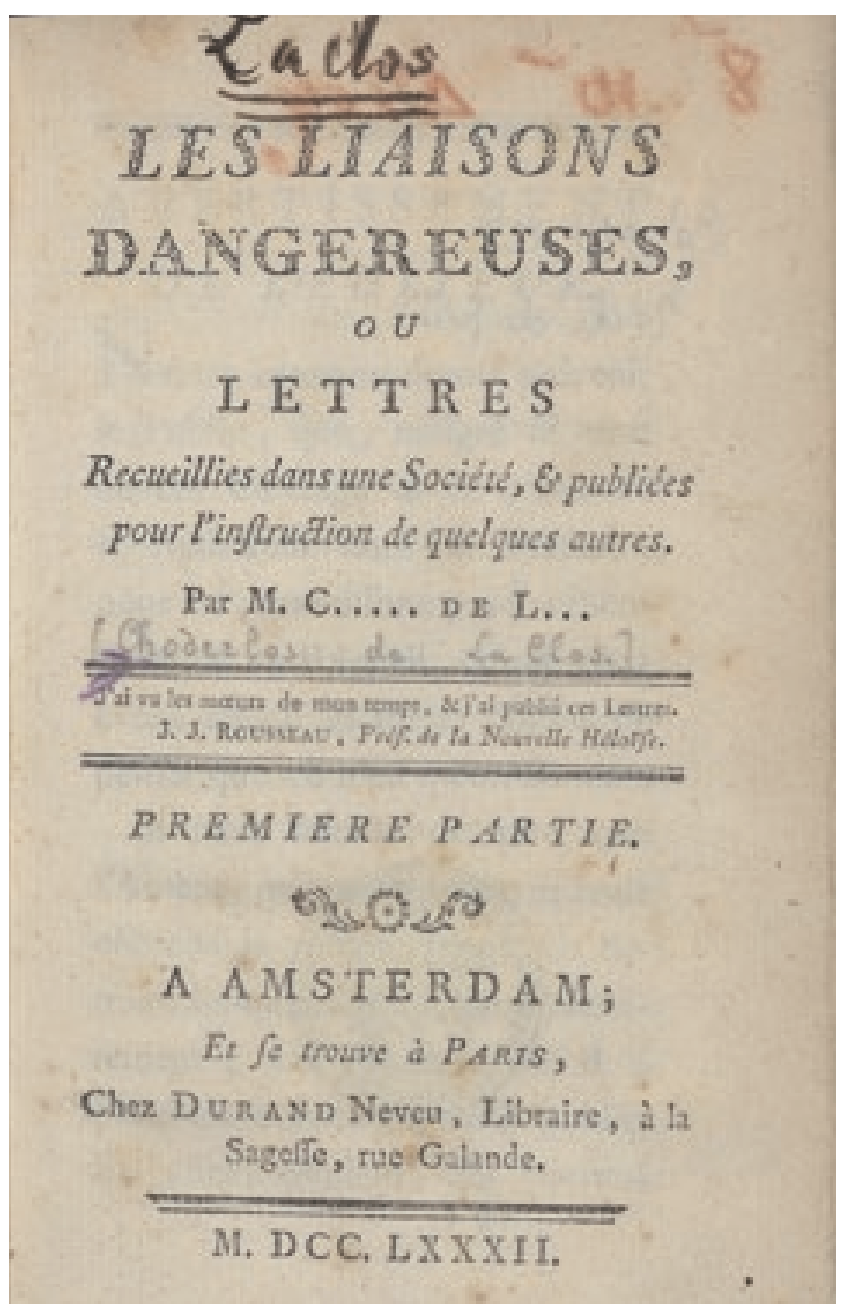

Page de titre de l'édition originale des Liaisons dangereuses de Choderlos de Laclos (Amsterdam, Durand neveu, 1782 ; coll. BNU) 


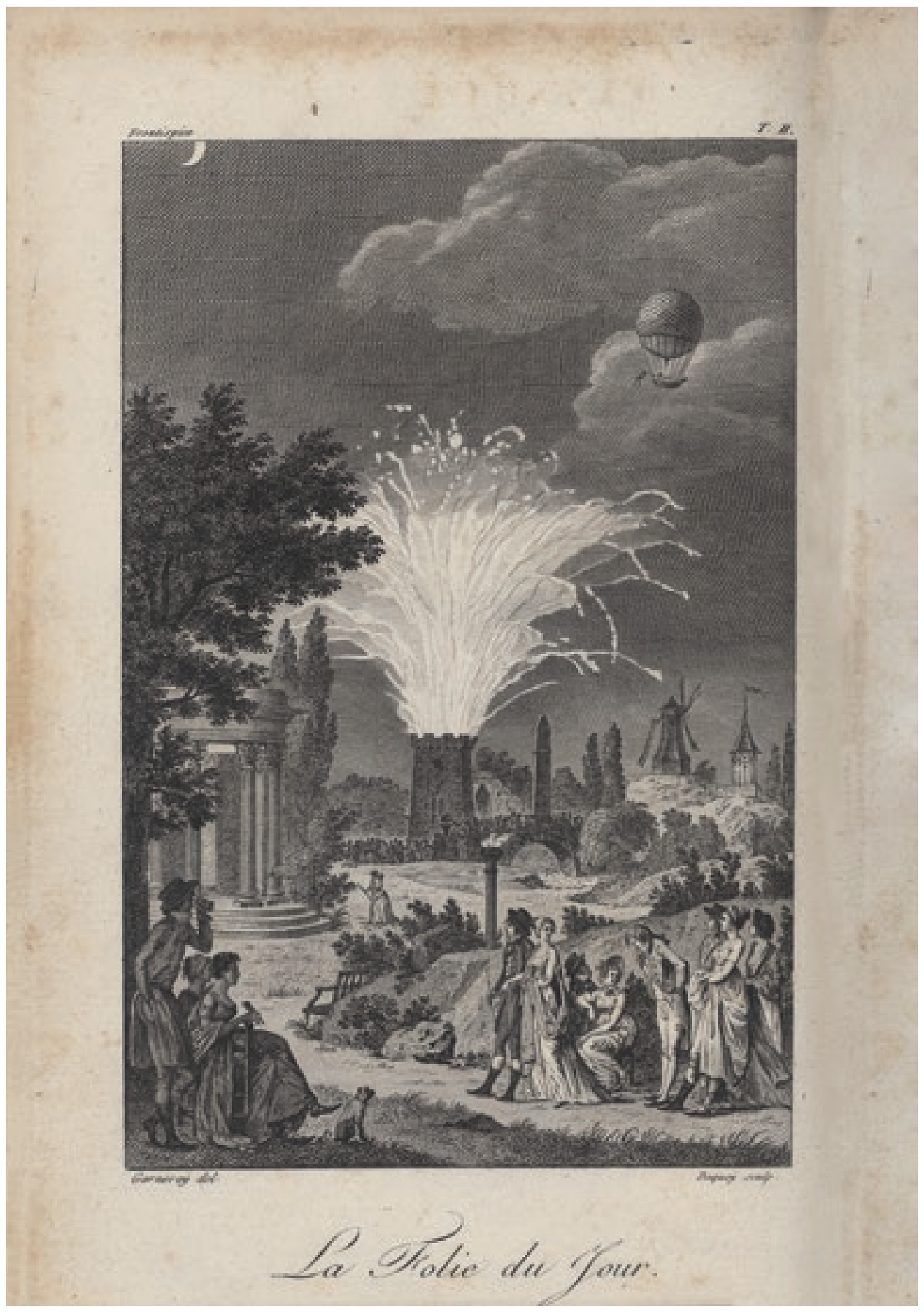

"La folie du jour ", gravure de Garnerey et Baquoy pour

Le Nouveau diable boiteux, tableau philosophique et moral de Paris (Paris, Buisson, 1798 ; coll. BNU) 
de Saint-Pierre. Enfin, pour la première partie du $19^{\mathrm{e}}$ siècle dont il était le contemporain, la bibliothèque de Johann Philipp offre un panorama presque complet des plus grands auteurs français, et notamment des romantiques : Hugo (une édition de 1852 de Napoléon le Petit et une édition de 1840 des Rayons et les Ombres), Chateaubriand (avec les éditions originales de plusieurs de ses opuscules politiques comme De la monarchie selon la Charte, 1816, ou Le roi est mort : vive le roi !, 1824), Dumas (édition originale de son drame Napoléon Bonaparte, ou Trente ans de l'histoire de France, 1831), Lamartine (éditions originales du Civilisateur, 1853, et des Confidences, 1849), Stendhal (édition originale du Rouge et le Noir, 1831 ${ }^{8}$ ), Mérimée, Sainte-Beuve, Nodier, Constant, Gautier (une édition d'un Trio de romans en 1852), les frères Goncourt, George Sand (deuxième édition du Compagnon du tour de France, 1841) ou Germaine de Staël (édition originale de De l'Allemagne, 1813).

Dans cette liste, on aura remarqué, à l'instar des deux derniers noms cités, de nombreuses femmes de lettres. Leurs œuvres témoignent, avec de nombreux autres ouvrages de sa bibliothèque, de la fascination - éminemment sympathique - de Johann Philipp pour le beau sexe. De ce point de vue aussi, ce n'est manifestement pas là la bibliothèque d'un diplomate et d'un politicien austère et aride ! On y trouve ainsi non seulement des ouvrages sur Madame Récamier, le Magasin des adolescentes de Jeanne-Marie LeprinceBeaumont (s. d.), les Femmes de la Révolution de Michelet (édition originale de 1854) et les Portraits de femmes de SainteBeuve (1852), mais aussi de très nombreux mémoires de femmes de cour et de femmes de lettres (outre celles déjà citées, la marquise de Créquy, la marquise de Lambert ou Julie de Lespinasse) ou de maîtresses d'hommes d'État (comme la tristement célèbre maîtresse de l'amiral Nelson, Lady Emma Hamilton, ou Louise de la Vallière, première maîtresse officielle de Louis XIV) - sans parler de plusieurs volumes de "Mémoires indiscrètes ". Certes, Johann Philipp était un catholique fervent. Mais cela n'empêche pas sa bibliothèque de recéler plusieurs ouvrages aux titres évocateurs, voire franchement libertins, comme La Religieuse de Diderot déjà mentionnée, Les Liaisons dangereuses de Laclos (édition originale de 1782), le roman de Vivant Denon Point de lendemain (édition de 1780), ou encore la Lettre d'une chanoinesse de Lisbonne à Melchior, officier français (1780), Les Vierges martyres (1846) ou le Dictionnaire d'amour de Catherine-Joseph-Ferdinand Girard de Propiac (1808)...
Ainsi se dessine peu à peu le profil d'une bibliothèque typique de ce que l'on pourrait appeler la culture européenne des humanités, teintée de l'esprit de liberté qui soufflait sur les Lumières françaises. Aux grands auteurs de la littérature s'ajoutent en effet ceux de la philosophie. Antique, d'abord, avec des œuvres de Platon (ses œuvres, traduites en latin par Marsile Ficin et publiées à Paris en 1522) et d'Aristote (sa Politique). Moderne ensuite, avec non seulement les Essais de Montaigne et des œuvres des philosophes français des Lumières déjà cités (notamment la deuxième édition de 1749 de L'Esprit des lois de Montesquieu ou la troisième édition de l'Essai sur le despotisme de Mirabeau, 1792), mais aussi des œuvres de Kant (l'édition originale allemande des Principes métaphysiques de la théorie du droit, 1797), de Hobbes (une édition de $1657 \mathrm{du}$ De Cive), de Tocqueville (De la démocratie en Amérique dans une édition de 1838), d'Edmund Burke, de Victor Cousin ou de Mendelssohn.

Enfin, on ne saurait clore ce tour d'horizon de la culture européenne sans évoquer les arts. Il est vrai que - si l'on excepte bien sûr les nombreux guides et récits de voyage, qui souvent évoquent des villes européennes, surtout italiennes (Venise, Rome, Florence, Naples, Milan, Vérone, etc.), riches en beautés artistiques - les beaux-arts sont relativement peu représentés dans la bibliothèque de mon aïeul. On remarquera tout au plus un Essai sur l'histoire de la peinture en Italie du célèbre comte Orloff (1823), un Essai sur les beaux-arts d'un certain Edme-François-Antoine-Marie Miel (1817) et une notice sur les tableaux du Musée royal de La Haye. En revanche, son goût pour la musique est manifeste. En témoignent, d'une part, les livrets et partitions d'opéra présents en nombre dans sa bibliothèque - de Rossini (Zelmira, Armide) à Mozart (Titus) en passant par des opéras comiques du $18^{e}$ siècle français (Jérôme et Fanchonnette de Jean-Joseph Vadé ou Sancho Pança dans son isle d'AntoineAlexandre-Henri Poinsinet) -, mais aussi et surtout les nombreuses partitions et méthodes pour guitare ou piano qui laissent entendre que de la musique devait résonner parfois dans les salles du château de Feldkirch.

$\mathrm{Au}$ total, la bibliothèque de mon quintaïeul me semble être non seulement celle d'un diplomate et d'un homme d'État, mais aussi plus largement celle d'un humaniste européen. Or que serait la culture des humanités sans les vertus de curiosité et d'étonnement qu'Aristote posait déjà comme fondement de la pensée ? Aussi voudrais-je terminer ce survol de la bibliothèque de Johann Philipp von Wessenberg en 
évoquant quelques " curiosités " qui s'y trouvent, des ouvrages inclassables dont le contenu ou le titre n'ont pas manqué, précisément, de piquer ma curiosité. Des ouvrages sur les mœurs et manières du " grand monde ", tout d'abord : L'Art d'obtenir des places, ou la Clef des ministères, ouvrage dédié aux gens sans emploi et aux solliciteurs de toutes les classes de Jacques-Gilbert Ymbert (1816), le Dictionnaire des girouettes, ou Nos contemporains peints d'après eux-mêmes d'Alexis Eymery (1815), les malicieuses caricatures de Daumier dans Le Musée pour rire (1840) et - cela est toujours utile en société - cette Instruction pour conserver les dents belles et saines, aux diverses époques de la vie de 1811. Des livres aux titres intrigants, ensuite, comme ce Nouveau diable boiteux, tableau philosophique et moral de Paris : mémoires mis en lumière et enrichis de notes par le Docteur Dicaculus, de Louvain (dû en réalité à Pierre-Jean-Baptiste Chaussard, en 1798), Dieu auteur des grands événemens arrivés en France en 1814 : par un député des Côtes-du-Nord, au Corps législatif, en l'an V (1814) et même une Histoire des chevaux célèbres, contenant un recueil des anecdotes relatives à ce noble animal... publiée par Pierre-Jean-Baptiste Nougaret en 1810. Et enfin, deux ouvrages qui pourraient être candidats au titre de "titre le plus rasoir ": de par sa longueur, l'œuvre d'un de mes compatriotes, un certain Mr. Madden, The infirmities of Genius illustrated by reffering the anomalies in the litterary character, to the habits and constitutional peculiarities on Men of Genius (1833) ; et pour la dimension pécuniaire et très pointue de son sujet - en dehors du fait que mes connaissances en économie sont très limitées - un ouvrage de l'écossais James Stuart qui m'a particulièrement frappé : The Principles of money applied to the present state of the coin of Bengal (1772).

Ainsi se termine mon exposé, non sans que je soulève auparavant pour finir quelques questions auxquelles je n'ai pas trouvé de réponse et que je laisse à ceux qui voudraient s'en saisir. Une " gender issue ", tout d'abord, autrement dit une question de genre : pourquoi devraiton partir du principe que la bibliothèque du château de Feldkirch, aujourd'hui à Strasbourg, n'appartenait qu'au seul Johann Philipp, et pas également à son épouse Marie-Gertrude ? Comment expliquer sinon la présence dans la bibliothèque de plusieurs volumes de la revue londonienne The Lady's Magazine? Ensuite, y a-t-il une raison particulière qui explique pourquoi l'inventaire alphabétique de sa bibliothèque reprend trois fois de suite au début, par la lettre " a " ? Est-ce lié au fait que le transfert de la bibliothèque à Strasbourg se fit en plusieurs livraisons ? Ou bien le signe que la bibliothèque de Feldkirch avait elle-même plusieurs parties, voire plusieurs utilisateurs ? Enfin, dans quelle mesure est-ce Johann Philipp lui-même qui a choisi seul les livres qui figurent dans sa bibliothèque et dans quelle mesure ce choix a-t-il été guidé par les nombreux volumes de catalogues de livres que celle-ci contient?

Quoi qu’il en soit des réponses à ces questions, espérons que ces quelques modestes remarques sur ce trésor livresque auront su éveiller la curiosité et le désir d'en savoir plus sur cette exceptionnelle bibliothèque.

\section{Roger Massie ${ }^{9}$}

(traduit, revu et augmenté par Julien Collonges) 


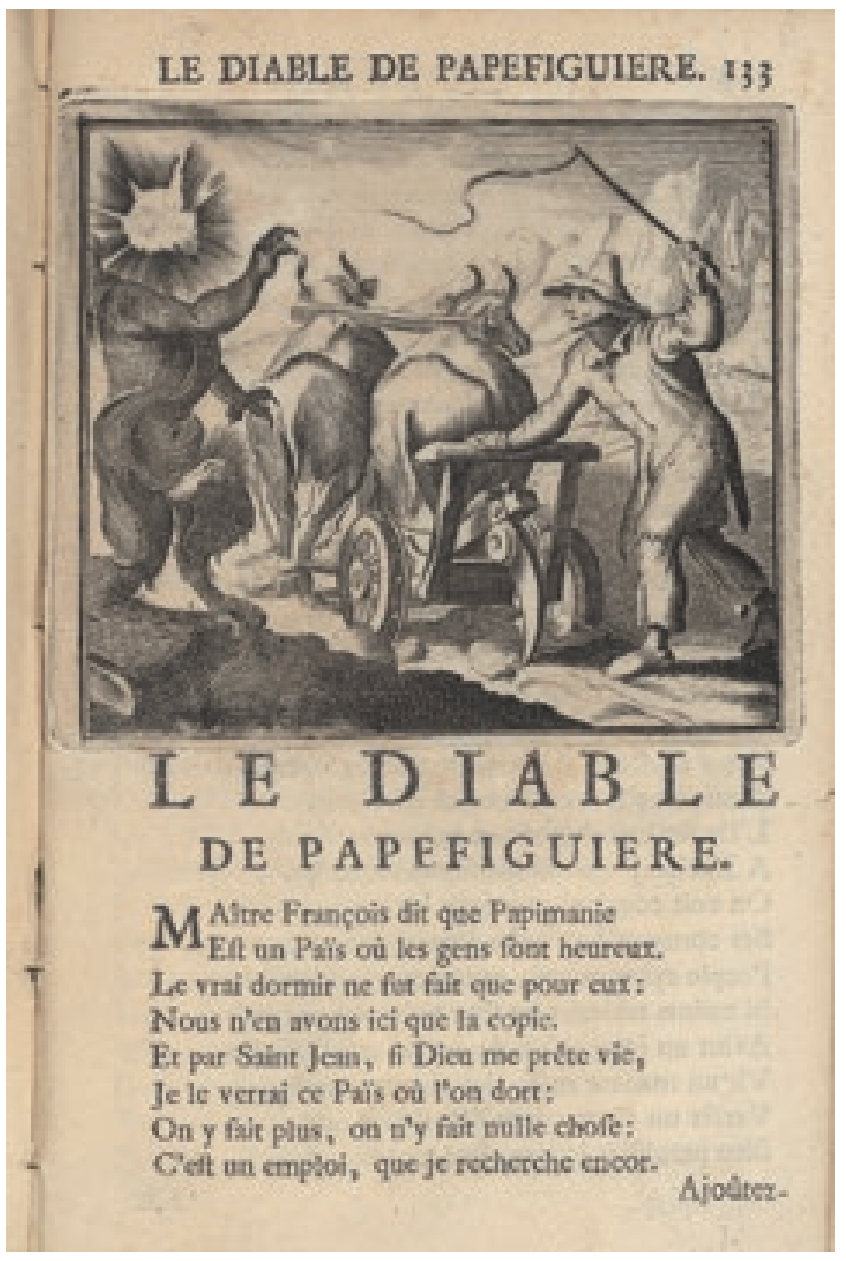

Gravure de Romain de Hooge pour

" Le Diable de Papefiguière " de Jean de La Fontaine (in Contes et nouvelles, Amsterdam, N. E. Lucas, 1721 ; coll. BNU)

\section{Notes}

1 - Pour de plus amples informations sur Johann Philipp von WessenbergAmpringen, voir la seule monographie (en allemand) qui lui a été consacrée : Alfred von Arneth, Johann Freiherr von Wessenberg: ein österreichischer Staatsmann des neunzehnten Jahrhunderts, Wien, W. Braumüller, 1898 ; pour une notice en français, voir Louis Gabriel Michaud (éd.), Biographie universelle ancienne et moderne, vol. 44, Leipzig, Brockhaus, $1843-1856$, p. 500 [note du traducteur].

2- Descendant direct de Johann Philipp von Wessenberg-Ampringen, Peter Heinrich von Wessenberg est notamment le fondateur de la Wessenberg-Akademie, une plateforme et un forum de discussion sur l'histoire des régions du Rhin et du Danube (www.wessenberg.at) [Note du traducteur].

3 - J'ai pu consulter ces archives en 2004 grâce à M. Gérard Littler, qui fut administrateur de la BNU de 1992 à 2002, puis, jusqu'à sa retraite, conservateur en charge des collections patrimoniales. Je souhaite lui exprimer ici encore ma gratitude et mentionner l'article qu'il a publié en 2002 dans le $n^{\circ} 4$ (vol. 47) du Bulletin des bibliothèques de France, sous le titre « Constitution de la collection dans la période allemande (18711918)". Mes remerciements s'adressent également à Monica Azulay Gaspard, qui m'a assisté dans l'étude de la bibliothèque de mon aïeul.

4- Voir la rubrique " L'inédit " du présent numéro, où une partie de ces Fragmens politiques est publiée pour la première fois.

5- Sur cet ouvrage, voir l'article d'Élise Girold intitulé "Saint-Simon, philosophe visionnaire et précurseur de l'Europe », publié sur le site internet de la BNU (www.bnu.fr) dans la livraison d'août 2015 de la rubrique " Trésor du mois " [note du traducteur]

6 - Effectivement signalé dans l'inventaire du don Wessenberg, et présent dans les " Registres noirs " qui contiennent le catalogue de la période allemande, ce volume a malheureusement disparu depuis des collections de la BNU [note du traducteur].

7 - Cette édition a été présentée récemment à la BNU dans le cadre de l'exposition Fénelon et son double (du 18 juin au 14 août 2015). Cf. le catalogue de l'exposition: Fénelon et son double, sous la direction de François-Xavier Cuche et Julien Gueslin, Strasbourg, Bibliothèque nationale et universitaire, 2015, p. 51 [note du traducteur].

8 - Cette édition a été présentée récemment à la BNU dans le cadre de l'exposition Métamorphoses : un bâtiment, des collections (du 11 avril au 20 septembre 2015). Voir l'analyse de cette édition originale proposée par Bertrand Marquer, maître de conférences à l'Université de Strasbourg, dans le catalogue de l'exposition : Métamorphoses : un bâtiment, des collections, sous la direction de Christophe Didier et Madeleine Zeller, Strasbourg, Bibliothèque nationale et universitaire, 2015, p. 268-269 [note du traducteur].

9 - Né en Inde et ancien fonctionnaire au Conseil de l'Europe, Roger Massie - qui réside aujourd'hui à Kolbsheim, en Alsace - est comme son cousin Peter Heinrich von Wessenberg le descendant de Johann Philipp von Wessenberg. Le texte publié ici est une version largement revue et augmentée d'une conférence donnée en 2004 avec le concours et les recherches diligentes de Mme Monica Azulay Gaspard, lors de la $3{ }^{\mathrm{e}}$ réunion annuelle de la Wessenberg-Akademie, à Liebenswiller, en Alsace [note du traducteur]. 